\title{
UWSF - von der Sprache über das Konzept zum neuen Titel
}

\author{
Henner Hollert • Almut B. Heinrich
}

Erhalten: 16. April 2010/Akzeptiert: 27. April 2010/Online veröffentlicht: 1. Juli 2010

(C) Springer-Verlag 2010

\section{UWSF wird englischsprachig}

Das Editorial der Juniausgabe (Heinrich 2010) befasste sich mit den Gründen, auch englischsprachige Beiträge in die bisher rein deutschsprachige UWSF aufzunehmen. Dies geschieht zum einen auf Grund der Leserumfrage (Heinrich und Hollert 2009), deren Ergebnis in diesem Punkt eindeutig war (Heinrich 2010), zum anderen auf Wunsch der Herausgeber und des Verlages, die sich so eine größere Bereitschaft der Autoren erhoffen, vor allem auch Originalarbeiten in UWSF zu publizieren; hinzu kommt der Wunsch, das hohe Potenzial von Springer für Vertriebswege vor allem auch außerhalb des deutschsprachigen Bereiches zu nutzen. Mit einem Wort, unsere Zeitschrift will und soll expandieren.

Zahlreiche Zeitschriften haben lange vor uns ihre Sprache auf Englisch umgestellt, auf die unterschiedlichste Weise. Einige haben übergangsweise beide Sprachen zugelassen, indem die deutschsprachigen Beiträge übersetzt wurden - z. B. bei „Fresenius' Zeitschrift für Analytische Chemie“ und „Lebensmittel-Untersuchung und -Forschung“, beides Springer Zeitschriften - um dann ganz auf Englisch überzugehen.

Hinzu kommt, dass eine Zeitschrift den Zeitströmen unterliegt, ja unterliegen muss, sonst wird sie von ihnen überrollt; und diese Zeitströme sind nun einmal angloamerikanisch orientiert.

H. Hollert

RWTH Aachen University,

Institute for Environmental Research (Biology V),

Department of Ecosystem Analysis,

Worringerweg 1, 52074 Aachen, Deutschland

A. B. Heinrich $(\triangle)$

Managing-Editor on behalf of Springer-Verlag,

Kirschgartenstr. 91, 69126 Heidelberg, Deutschland

E-Mail: ABH.scientificjournals@googlemail.com

\section{UWSF wird zu einer europäischen Zeitschrift}

Mit der Sprache allein ist es jedoch nicht getan. Eine Sprachänderung ist immer auch ein Signal, das Konzept der Zeitschrift den genannten Zeitströmen anzupassen und konsequenterweise mit dem modifizierten Konzept auch den Titel zu ändern.

Wie im Editorial der Juniausgabe (Heinrich 2010) berichtet, wollte Otto Hutzinger, Gründer und langjähriger erster Herausgeber der UWSF, zwar eine deutschsprachige Zeitschrift gründen, trug sich aber auch mit dem Gedanken einer europäischen Zeitschrift (in allen relevanten Sprachen). Wir wollen jetzt nicht so weit gehen, dass Hutzinger der UWSF die Anlage mitgegeben hat, sich zu einer europäischen Zeitschrift zu entwickeln; aber wir können seine Weitsicht bewundern, die auf der Höhe seines visionären Schaffensdranges so charakteristisch für ihn war.

In der Tat haben wir jetzt, mit der Öffnung für englische Beiträge, die Möglichkeit, unsere Zeitschrift über den deutschsprachigen Bereich hinaus nach Europa zu orientieren. Auch das liegt (schon lange) im Trend der Zeit, wenn wir daran denken, dass nationales Recht nach einer Übergangszeit EU-Recht werden muss(te). Bei der „Anpassung“ ist es jedoch nicht geblieben, wie die folgenden Beispiele zeigen:

\subsection{Pflanzenschutzmittel-Richtlinie}

\subsubsection{Die ,alte“ Pflanzenschutzmittel-Richtlinie}

\section{EU 2004:}

Europäische Union (EU): Richtlinie des Rates vom 15. Juli 1991 über das Inverkehrbringen von Pflanzenschutzmitteln 91/414/EWG. Version vom 1.1.2004, S. 1-209 


\subsubsection{Die „,neue“ Pflanzenschutzmittel-Richtlinie}

EU 2009:

European Union: Regulation (EC) No 1107/2009 concerning the placing of plant protection products on the market and repealing (= aufheben) Council Directives 79/117/EEC and 91/414/EEC

2010 wird auch die Bewertungsphilosophie der Pflanzenschutzrichtlinie umgestellt von „Risk“ auf „Hazard“.

\subsection{REACH}

Die Verordnung (EG) Nr. 1907/2006 (REACH-Verordnung) ist eine EU-Chemikalienverordnung, die am 1. Juni 2007 in Kraft getreten ist. REACH steht für Registration, Evaluation, Authorisation and Restriction of Chemicals, also für die Registrierung, Bewertung, Zulassung und Beschränkung von Chemikalien. Als EU-Verordnung besitzt REACH gleichermaßen und unmittelbar in allen Mitgliedstaaten Gültigkeit. Durch REACH wird das bisherige Chemikalienrecht grundlegend harmonisiert und vereinfacht.

„Bei den gestaffelten Testsystemen wird die jährlich produzierte Menge als Trigger für komplexere Tests und bessere Daten eingesetzt, wie im Chemikaliengesetz vorgesehen. Die neue Europäische Chemikaliengesetzgebung $\mathrm{REACH}^{1}$ versucht, die künstliche Trennung in alte und neue Stoffe zu überwinden, um so auch viele Tausende von ungetesteten und unbewerteten Chemikalien einer Risikoanalyse zu unterziehen und ggf. Maßnahmen daraus abzuleiten. REACH bezieht sich auf die ca. 100000 im Register EINECS aufgeführten Industriechemikalien (darunter ca. 30000 Chemikalien im engeren Sinne). Chemikalien für spezielle Verwendungszwecke (Pestizide/ PSM-Wirkstoffe ${ }^{2}$, Biozide, Arzneimittelwirkstoffe) sind gesetzlich gesondert geregelt, wobei die ersten beiden Gruppen in Hinblick auf ihr Verhalten in der Umwelt geprüft werden müssen, während die Umweltbewertung bei den Arzneimitteln und deren Metaboliten (die vor allem in den Klärwerken und deren Vorflutern in wachsender Anzahl nachgewiesen werden) noch in den Kinderschuhen steckt" (Klöpffer, pers. Mitt.). Umweltrecht (in Europa) ist europäisches Recht.

\subsection{Europäische Wasserrahmenrichtlinie}

Auch die Gewässerschutzpolitik der europäischen Gemeinschaft hat seit Ende 2000 ein neues Fundament: die Richtlinie 2000/60/EG, mit vollständigem Namen „Richtlinie des

${ }^{1}$ REACH 2006.

${ }^{2}$ EU 2004, 2009.
Rates zur Schaffung eines Ordnungsrahmens für Maßnahmen der Gemeinschaft im Bereich der Wasserpolitik“, kurz Wasserrahmenrichtlinie (WRRL). Durch Ersatz, Zusammenfügung und Neugestaltung der seit den 1970er-Jahren verabschiedeten Elemente europäischer Gewässerschutzpolitik ist es gelungen, ein konsistentes, umfassendes und transparentes Konzept aufzustellen, das in den kommenden Jahrzehnten das wasserwirtschaftliche Handeln in Europa bestimmen wird.

Die WRRL verfolgt einen ganzheitlichen Ansatz für einen integrierten Gewässerschutz. Sie setzt anspruchsvolle Ziele, insbesondere bezüglich der Erreichung eines weitgehend ökologisch ausgerichteten „guten Zustandes“ der Gewässer und gibt die wesentlichen Prozesse, Instrumente sowie einen klaren Zeitplan auf dem Weg zu diesem Ziel vor (Hollert et al. 2007). Die Umsetzung der europäischen WRRL geschieht durch nationale bzw. europäische Bewirtschaftungspläne. Auch dies dokumentiert die engen Verflechtungen zwischen europäischem und nationalem Recht.

Zum EU-Projekt MODELKEY (Hein et al. 2010), welches der WRRL zuarbeitet (ein Beitrag der Juniausgabe) siehe unten.

In einer der folgenden Ausgaben wird das EU Life+Projekt $\mathrm{M}^{3}$ vorgestellt, welches durch eine Partnerschaft bestehend aus dem Erftverband, dem Waterboard Delfland in den Niederlanden und dem Centre de Recherche Public Henri Tudor in Luxemburg - Beiträge zu den Programs of Measures (POM) der WRRL liefern möchte. Der Beitrag zeigt Defizite der aktuellen Monitoringansätze im Rahmen der WRRL auf (Galle et al. 2010, in Vorbereitung).

\section{Umweltrecht und Umweltwissenschaft}

Umweltrecht ist eng verflochten mit Umweltwissenschaft. Im Editorial der Juniausgabe wird gezeigt, dass Henner Hollert (der Nachfolger von Otto Hutzinger) das enge Miteinander von wissenschaftlichen und regulatorischen Aspekten als grundlegend ansieht (Heinrich 2010). Wir erinnern uns an den jahrelangen Appell (der auch in UWSF seinen Niederschlag fand) an die Wissenschaft, die regulatorischen Gremien zu beraten, und zwar fundiert, aber in verständlicher Sprache; denn es herrschte lange Zeit Sprachlosigkeit zwischen den beiden Bereichen. Inzwischen ist erkannt worden, dass zwischen ihnen ein Miteinander, eine Symbiose bestehen sollte und bestehen muss (siehe auch unsere Beitragsreihe „Regulatorische Ökotoxikologie").

Die Schlüsselwörter der obigen Ausführungen lauten:

- Europa,

- Umweltrecht/Umweltpolitik und

- Umweltwissenschaft. 
Daraus ergibt sich das folgende (modifizierte) künftige Konzept der Zeitschrift:

1. Die ,neue“ UWSF will ein Sprachrohr sein nach Europa und umgekehrt aus Europa berichten. Damit verbindet sie den regionalen und europäischen Bereich.

2. Sie will den regionalen und europäischen Bereich nicht nur verbinden, sondern vor allem auch deren Interaktion beleuchten.

3. Sie will Umweltwissenschaft und Umweltrecht/Umweltpolitik fokussieren.

Die Verbindung von Wissenschaftlern und Entscheidungsträgern auf regionaler und europäischer Ebene im Umweltbereich betrachten wir als den Kernpunkt des künftigen Profils.

Die drei englischen Beiträge in der Juniausgabe interpretieren dieses Konzept deutlich:

Hofmann et al. (2010) sowie Wurbs et al. (2010) beschreiben eine Methode, die wissenschaftliche Aspekte mit regulatorischen Maßnahmen innerhalb der EU verbindet. Darüberhinaus wurde die von Hofmann et al. (2010) entwickelte Methode von CEN (European Committee for Standardization) zum europäischen Standard erklärt (Interaktion).

Hein et al. (2010) konnten in einem Beitrag über das integrierte EU-Projekt MODELKEY zeigen, welche Elemente für das Erreichen des guten ökologischen Zustandes (das erklärte Ziel der EU Water Framework Directive, siehe WRRL, Abschn. 2.3) in ausgewählten europäischen Flusseinzugsgebieten zusätzlich in die Bewirtschaftungspläne integriert werden sollen.

Über dieses Konzept hinaus werden beibehalten:

- Das 3-Säulen-Prinzip (Hochschule - Industrie - Behörde) innerhalb der Zielgruppen. ${ }^{3}$

- Die Beitragsarten: hier ändert sich nichts (vgl. Hollert 2006), es sei denn, sie werden erweitert. Publiziert werden: Research articles (including Short communications), Review articles, Series, Case studies, Discussion articles, Commentaries, Book reviews and announcements, Editorials, Letters to the Editor, Conference announcements and reports, Presentations (projects, institutions, curricula), News from policy and legislation, Corners. Aufnehmen sollten wir lt. Leserumfrage: Pro \& Contra, New theses (summaries).

- Die Themenbreite; so wird z. B. der Bereich „Umweltchemie" und „Ökotoxikologie“ immer Bestandteil von UWSF bleiben, zumal auch die GDCh-FG, der UWSF verbunden ist, „Umweltchemie und Ökotoxikologie“ heißt. Auch die mit der UWSF assoziierte Society of Environmental Toxicology and Chemistry Europe - German

\footnotetext{
${ }^{3}$ Auch SETAC Europe bzw. SETAC Europe (German Language Branch), die Gesellschaft, die mit UWSF assoziiert ist, vertritt dieses Prinzip und hat es sogar in ihren Mitgliederstatuten fest integriert.
}

Language Branch e. V. - kurz SETAC GLB - hat Umwelttoxikologie und -chemie im Fokus.

\section{Vom erweiterten Konzept zum neuen Titel}

Es wäre kontraproduktiv, wollten wir den bisherigen Titel „Umweltwissenschaften und Schadstoff-Forschung" beibehalten. Zum einen wird er von englischsprachigen Lesern und Autoren nicht verstanden; zum anderen hat ein neuer Titel Signalwirkung: Seht, hier hat eine neue Entwicklung stattgefunden.

Vom Konzept zum Titel ist es nur ein kleiner Schritt. Betrachten wir noch einmal die Schlüsselwörter:

- Europe,

- environmental regulation und

- environmental science.

Daraus ergibt sich die folgende Titelformulierung:

Environmental Sciences Europe

Bridging Science and Regulation at the Regional and European Level

\section{Der internationale Bereich}

Wenn auch der künftige Schwerpunkt der Zeitschrift „Europa“ heißt, so kann der internationale Bereich nicht ausgeklammert werden, denn Wissenschaft ist international. Im regulatorischen Bereich sieht das anders aus, wie REACH und TOSCA ${ }^{4}$ zeigen.

Dennoch sehen wir aufgrund des unterschiedlichen Konzeptes keine Nähe zur ESPR (Environmental Science and Pollution Research).

\section{Literatur}

Hein M et al. (2010) MODELKEY - Key findings and recommendations for reaching the EU Water Framework Directive's quality objectives. Umweltwiss Schadst Forsch 22(3). doi:10.1007/ s12302-010-0137-2

Heinrich AB (2010) Publish - in English - or perish. Umweltwiss Schadst Forsch 22(3). doi:10.1007/s12302-010-0132-7

Heinrich AB, Hollert H (2009) Zur zielgruppengerechten Gestaltung der UWSF. Umweltwiss Schadst Forsch 21(5):417-418

Hofmann F et al. (2010) Monitoring of Bt-Maize pollen exposure in the vicinity of the nature reserve Ruhlsdorfer Bruch in northeast Germany 2007 to 2008. Umweltwiss Schadst Forsch 22(3). doi:10.1007/s12302-010-0133-6

Hollert H, Heise S, Keiter S, Heininger P, Förstner U (2007) Wasserrahmenrichtlinie - Fortschritte und Defizite. Umweltwiss Schadst Forsch 19, Sonderheft Nr. 1:58-70

\footnotetext{
${ }^{4}$ In den USA soll TSCA, „Toxic Substances Control Act“ (auch TOSCA genannt), eines der ersten Chemikaliengesetze (es trat am 1. Januar 1977 in Kraft) novelliert werden.
} 
Hollert H (2006) Der neue Herausgeber stellt sich vor: Umweltwissenschaften und Schadstoff-Forschung - interdisziplinäre Zeitschrift für Umweltchemie und Ökotoxikologie im Kontext. Umweltwiss Schadst Forsch 18(1):1-2
Wurbs A et al. (2010) Regionalisation of flora elements in field boundaries sensitive to hybridisation with genetically modified oilseed rape. Umweltwiss Schadst Forsch 22(3). doi:10.1007/s12302010-0134-5 